\title{
ROTURA ESPONTÁNEA DE ÚTERO BICORNE EN GESTACIÓN ASOCIADA A ACREIISMO PLACENTARIO
}

\author{
Mónica Novelle García ${ }^{1}$, Ricardo Savirón Cornudella², Paula Suárez Mansilla , Ginés \\ Hernández Cortés ${ }^{1}$, Ramón Usandizaga Elio ${ }^{1}$ \\ ${ }^{1}$ Servicio de Ginecología y Obstetricia, Hospital Universitario La Paz, Madrid, España. ${ }^{2}$ Servicio de Ginecología y \\ Obstetricia, Hospital Universitario Puerta de Hierro, Madrid, España.
}

\section{RESUMEN}

La rotura espontánea en útero bicorne gestante es un accidente poco frecuente y difícil de diagnosticar. La prevalencia de malformaciones uterinas congénitas entre las mujeres fértiles está documentada entre 1-2\% de la población; de éstas, el útero bicorne varía entre un 25 a 37\%. Generalmente la rotura de la pared uterina sucede en el segundo trimestre, presentándose como intenso dolor abdominal a consecuencia de la hemorragia intraperitoneal. Presentamos caso clínico de una paciente gestante de 19 semanas con útero bicorne en el que se produjo una rotura uterina asociada a acretismo placentario. La placenta acreta y el útero bicorne son 2 entidades muy poco frecuentes pero que pueden asociarse. Como tratamiento se realizó una hemihisterectomía izquierda con una evolución satisfactoria.

\section{PALABRAS CLAVES: Útero bicorne, rotura uterina, acretismo placentario}

\section{SUMMARY}

The sudden rupture in bicornuate pregnant uterus is an uncommon and difficult-to-diagnose occurrence. The prevalence of congenital uterine malformations amongst fertile women is documented for $1-2 \%$ of the population; of these, bicornuate uterus varies from 25 to $37 \%$. The rupture of the uterine wall happens generally in the second trimester, accompanied by an intense abdominal pain as a result of the intraperitoneal hemorrhage. We report a case of a 19 weeks pregnancy with an uterine rupture in a bicornuate uterus associated with a placental accretism. Both placental accretism and bicornuate uterus are uncommon but can be related. A left-side hemihysterectomy was applied leading to a satisfactory evolution.

\section{KEY WORDS: Bicornuate uterus, uterine rupture, placental accretism}

\section{INTRODUCCIÓN}

La rotura espontánea del útero bicorne durante el embarazo es un accidente poco frecuente y de difícil diagnóstico.

La prevalencia de malformaciones uterinas congénitas en mujeres fértiles está documentada entre el 1 a $2 \%$ de la población (1). De éstas, la más frecuente es el útero septo (2-4). La preva- lencia del útero bicorne varía en función de la bibliografía consultada entre un $25 \%$ y $37 \%$ de las malformaciones congénitas uterinas, mientras que el útero unicorne con cuerno rudimentario es más raro teniendo una frecuencia de 1:100.000 $(3,5,6)$.

Generalmente, la rotura de la pared uterina, en estas malformaciones, sucede en el segundo trimestre, presentándose como un intenso dolor ab- 
dominal a consecuencia de la hemorragia intraperitoneal (7).

Presentamos un caso clínico de una paciente gestante de 19 semanas con útero bicorne, ya conocido, en el que se produjo una rotura uterina asociada a acretismo placentario.

\section{CASO CLÍNICO}

Paciente de 31 años, secundigesta, gestante de $19+3$ semanas de amenorrea, que no presentaba antecedentes personales ni familiares de interés y entre sus antecedentes gineco-obstétricos destaca el antecedente de útero bicorne, con gestación anterior sin incidencias, con un parto a término mediante fórceps. La gestación actual presentaba una evolución dentro de la normalidad salvo por un episodio de pielonefritis que requirió hospitalización para observación y tratamiento antibiótico.

Consulta de urgencia por dolor abdominal intenso, a la exploración ginecológica se objetivó un cérvix único sin modificaciones, ausencia de metrorragia, abdomen ligeramente distendido con defensa y doloroso a la palpación de forma generalizada. La paciente presentaba discreta palidez cutáneo-mucosa, frialdad, hipotensión e hipotermia. Se realiza una ecografía donde se visualiza un feto en situación transversa con ausencia de latidos cardiofetales, biometría acorde a 18-19 semanas, presencia de líquido libre en moderada cantidad en cavidad abdominal.

Se ingresa a la paciente y se procede a la realización de analíticas sanguíneas seriadas observándose un descenso progresivo del hematocrito y la hemoglobina desde un $32 \%$ a $25 \%$ y de $11,4 \mathrm{~g} / \mathrm{dl}$ a $9,2 \mathrm{~g} / \mathrm{dl}$, respectivamente, en 50 minu-

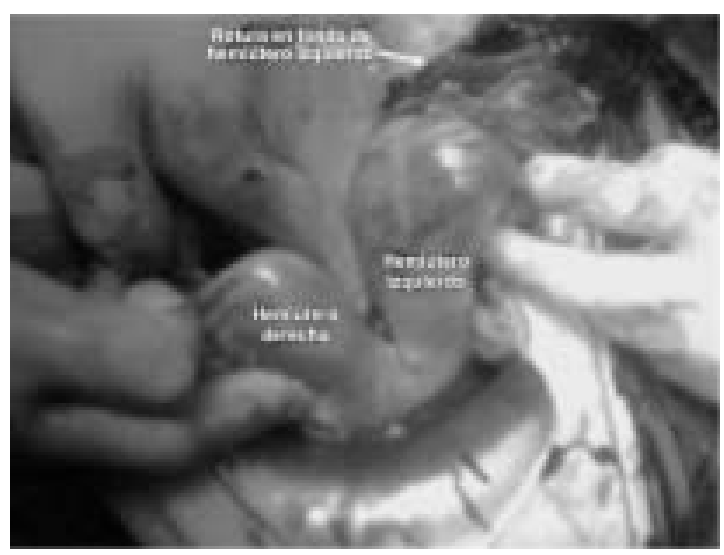

Figura 1. Rotura del hemiútero izquierdo a nivel de fondo uterino. tos, junto con pruebas de coagulación normales y 30.000 leucocitos/dl. En vista del deterioro progresivo de la paciente se decide realizar una laparotomía exploradora.

En quirófano se transfunden 4 concentrados de glóbulos rojos. Se observa la rotura del hemiútero izquierdo a nivel de fondo uterino (Figura 1) encontrándose la gestación con bolsa íntegra libre en cavidad abdominal junto con hemoperitoneo. Frente a estos hallazgos se procede a la aspiración del hemoperitoneo y posterior realización de una hemihisterectomía izquierda (Figura 2 y 3). La paciente tuvo una evolución postoperatoria favorable y fue dada de alta con ferroterapia al tercer día. El informe anatomopatológico describe malformación uterina, segmento uterino con cambios sugestivos de acretismo placentario

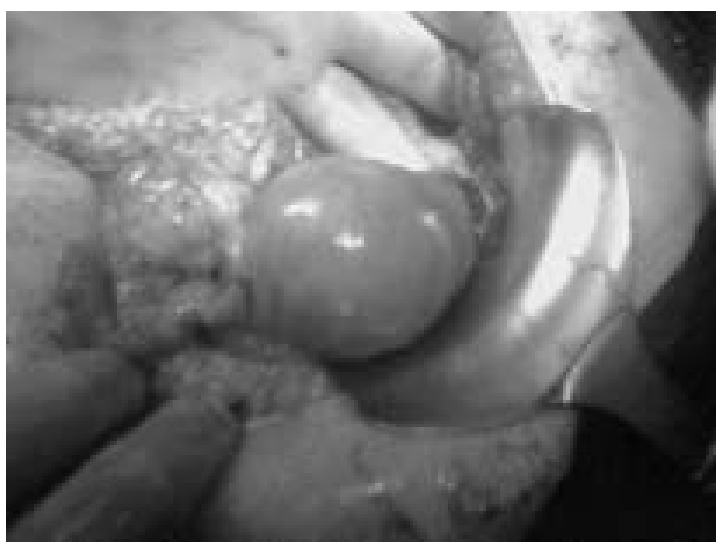

Figura 2. Hemihisterectomía izquierda.

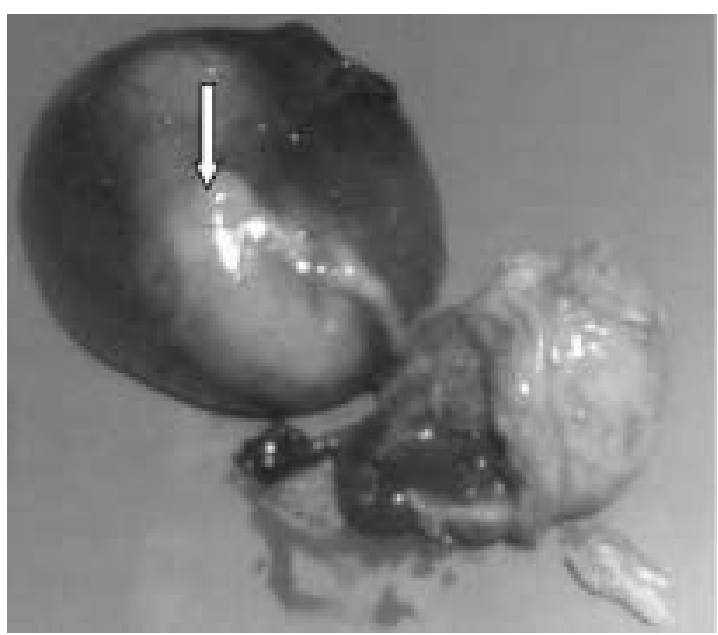

Figura 3. Pieza de hemihisterectomía izquierda y feto incluido en bolsa amniótica (flecha). 
y placenta inmadura con extensa necrosis isquémica.

\section{DISCUSIÓN}

Las malformaciones uterinas son el resultado de una interrupción en el desarrollo, una formación anormal o una fusión incompleta de los conductos mesonéfricos. Estas anomalías son raras siendo el útero septo la más frecuente, representando el $80 \%$ de casos de malformaciones uterinas mullerianas (8). La clasificación más utilizada es la de la Sociedad Americana de Fertilidad (AFS), donde el útero bicorne está incluido en el grupo IV y el unicorne en el grupo II, entre los cuales habría que realizar el diagnóstico diferencial principalmente.

Dichas malformaciones han sido relacionadas con una elevada tasa de infertilidad en forma de abortos recurrentes, prematuridad y otras complicaciones obstétricas, aunque en muchas pacientes pueden permanecer asintomáticas $(7,9-12)$. Se asocian con cierta frecuencia a otras anomalías del desarrollo, particularmente del tracto urinario $(31 \%)$, siendo la más común la agenesia renal unilateral, por lo que se recomienda en estas pacientes hacer un estudio urológico exhaustivo (13). En nuestro caso este estudio no se ha llevado a cabo aunque hubiese sido recomendable ya que la paciente había presentado un episodio de pielonefritis que requirió hospitalización y que podría estar en relación con alguna alteración subyacente.

La rotura uterina es un problema obstétrico de gran riesgo vital. Dicha rotura suele suceder en primigrávidas en el primer o en el segundo trimestre asociado a malformaciones uterinas como el útero unicorne o bicorne. La particularidad de nuestro caso radica en que la paciente no era primigrávida sino que previamente tenía una gestación de curso normal y con un parto a término mediante fórceps. Casos similares fueron publicados por Mane y cols (14) y Kore y cols (15).

El diagnóstico de una rotura uterina en un útero malformado es, en ocasiones, muy complicado como hemos podido observar en la revisión bibliográfica, ya que en las pruebas de imagen no se observa claramente la rotura uterina o esta se produce más tarde, la analítica sanguínea puede tardar en revelar una pérdida hemática y la clínica en un principio puede ser muy anodina. Incluso se puede pensar inicialmente en un abruptio placentae como diagnóstico diferencial, sobre todo si la malformación no está diagnosticada previamente.

La ultrasonografía pueden ser de ayuda en el diagnóstico de estas anomalías antes de la rotura, lo que ayudaría a reducir la mortalidad y la morbilidad asociada al hemoperitoneo masivo que sucede durante la misma (16). Actualmente la resonancia magnética y la ecografía 3D están siendo de gran utilidad para diagnosticar malformaciones uterinas $(17,18)$ aunque por el momento no existe demasiada experiencia en dicho campo.

En nuestro caso, la ecografía que se realizó en urgencias no ponía claramente de manifiesto la malformación ni la rotura uterina, ya que sólo nos permitía discernir la existencia de un discreto hemoperitoneo y el fallecimiento del feto; además, la analítica sanguínea inicial del ingreso tampoco revelaba una pérdida hemática relevante. Se sospechó una rotura uterina por la evolución clínica y las analíticas seriadas que sugerían un sangrado progresivo. El diagnóstico definitivo se realizó tras una laparotomía exploradora.

Nuestro caso también estaba asociado a acretismo placentario, como se demostró en el estudio antomopatológico, lo que hemos podido observar también en otras series publicadas, y que, aunque no sea condición imprescindible para la rotura uterina, probablemente la favorezca. La placenta acreta y el útero bicorne son dos trastornos muy poco frecuentes pero que pueden asociarse, actuando la anomalía mulleriana como factor de riesgo poco usual de acretismo placentario (13).

La delgadez del músculo del cuerno rudimentario hace que tienda a la rotura entre la semana 10 y $15(19,20)$. En este caso, la paciente conocía previamente la malformación que presentaba, era una gestante de 19 semanas y se trataba de un útero bicorne completo lo que correspondería a una malformación tipo IV de la Clasificación de la Sociedad Americana de Fertilidad (8).

El tratamiento generalmente implica la extirpación del hemiútero que ha sufrido la rotura, como en nuestro caso, en el que se realizó una hemihisterectomía. Esto deja una cicatriz uterina, por lo que es recomendable evitar la gestación al menos durante un año. Además, futuras gestaciones requieren monitorización estrecha, hospitalización temprana y cesárea electiva al término (15).

A pesar de la escasa frecuencia del caso clínico presentado, creemos que conviene tenerlo presente en el diagnóstico diferencial de gestantes con dolor abdominal en el segundo trimestre, sobre todo si consta en sus antecedentes la presencia de una malformación uterina. 


\section{BIBLIOGRAFÍA}

1. Balasch J. In: Masson-Salvat, editor. Casos Clínicos. Obstetricia y Ginecología. Barcelona: MassonSalvat, 1990:548-55.

2. Cayuela E, Cararach M, Gilabert J, Pérez T, Rivero B, Torrejón R. In: Meditex, editor. Documentos de consenso de la SEGO. Madrid: 1996:21-2.

3. Heinonen PK. Unicornuate uterus and rudimentary horn. Fertil Steril 1997;68(2):224-30.

4. Handa Y, Hoshi N, Yamada H, Wada S, Kudo M, Tsuda $\mathrm{K}$, et al. Tubal pregnancy in a unicornuate uterus with rudimentary horn: a case report. Fertil Steril 1999; 72(2):354-6.

5. Sinha R, Mahajan C, Hegde A, Shukla A. Laparoscopic metroplasty for bicornuate uterus. J Minim Invasive Gynecol 2006;13(1):70-3.

6. Speroff L, Glass RH, Kase NG. El útero. En: Endocrinología Ginecológica e Infertilidad. Waverly Hispánica S.A. Buenos Aires, Argentina, Madrid, España: 2000: 123-158.

7. Daskalakis G, Pilalis A, Lykeridou K, Antsaklis A. Rupture of noncommunicating rudimentary uterine horn pregnancy. Obstet Gynecol 2002;100(5 Pt 2):1108-10.

8. The American Fertility Society classifications of adnexal adhesions, distal tubal occlusion, tubal occlusion secondary to tubal ligation, tubal pregnancies, mullerian anomalies and intrauterine adhesions. Fertil Steril 1988;49(6):944-55.

9. Golan A, Langer R, Bukovsky I, Caspi E. Congenital anomalies of the mullerian system. Fertil Steril 1989;51(5):747-55.

10. Green LK, Harris RE. Uterine anomalies. Frequency of diagnosis and associated obstetric complications. Obstet Gynecol 1976;47(4):427-9.

11. Raga F, Bauset C, Remohi J, Bonilla-Musoles F, Simon C, Pellicer A. Reproductive impact of conge- nital Mullerian anomalies. Hum Reprod 1997;12(10): 2277-81.

12. Heinonen PK, Saarikoski S, Pystynen P. Reproductive performance of women with uterine anomalies. An evaluation of 182 cases. Acta Obstet Gynecol Scand 1982;61(2):157-62.

13. Heredia AJ, Lois MM, Medrano P. Spontaneous uterine rupture associated with placenta accreta and uterus bicornate. Progresos en Obstetricia y Ginecología 2001;44:497-500.

14. Mane S, Chaudhry R, Nandanwar Y. An unusual presentation of pregnancy in bicornuate uterus. J Obstet Gynecol, India 1994; 44-1:154-5.

15. Kore S, Pandole A, Akolekar R, Vaidya N, Ambiye VR. Rupture of left horn of bicornuate uterus at twenty weeks of gestation. J Postgrad Med 2000; 46(1):39-40.

16. Achiron R, Tadmor O, Kamar R, Aboulafia Y, Diamant $Y$. Prerupture ultrasound diagnosis of interstitial and rudimentary uterine horn pregnancy in second trimester. A report of two cases. J Reprod Med 1992;37:89-92.

17. Amsalem $\mathrm{H}$, Yagel $\mathrm{S}$. Three-dimensional sonography of a fetus and an intrauterine device in a bicornuate uterus. J Ultrasound Med 2005;24(2):254.

18. Tsafrir A, Rojansky N, Sela HY, Gomori JM, Nadjari M. Rudimentary horn pregnancy: first-trimester prerupture sonographic diagnosis and confirmation by magnetic resonance imaging. $\mathrm{J}$ Ultrasound Med 2005;24(2):219-23.

19. Tang R, Sheng Y, Chen ZJ. Rupture of pregnancy in a communicating rudimentary uterine horn after in vitro fertilization and embryo transfer. Int J Gynaecol Obstet 2004;86(3):394-5.

20. Elsayegh A, Nwosu EC. Rupture of pregnancy in the communicating rudimentary uterine horn at 34 weeks. Hum Reprod 1998;13(12):3566-8. 